\title{
Composição florística e faunística de quintais agroflorestais da agricultura familiar no nordeste paraense
}

\section{Floristic and faunal composition homegardens of family farming in northeast Pará}

\author{
Amanda Paiva Quaresma ${ }^{1}$, Ruth Helena Cristo Almeida ${ }^{2}$, Cyntia Meireles de Oliveira ${ }^{3}$, Osvaldo Ryohei Kato ${ }^{4}$
}

Resumo: Os quintais agroflorestais são comuns entre os agricultores familiares da Amazônia, sendo sistemas de uso da terra sustentáveis desenvolvidos a partir dos saberes tradicionais. Além de ter como principal finalidade a promoção da segurança alimentar, são espaços de socialização e lazer para os agricultores e, podem contribuir para a composição de sua renda pela venda do excedente da produção. O objetivo desse trabalho foi identificar a diversidade dos quintais, a partir da composição de flora e fauna. A pesquisa estudou 37 quintais agroflorestais em cinco comunidades em Igarapé-Açu e Marapanim, nordeste paraense, usando técnicas de observação direta em campo, aplicação de questionários semiestruturados, notas etnográficas e turnê guiada nos quintais agroflorestais. Foram 107 espécies vegetais identificadas e 12 animais. Estes dados revelam que há uma quantidade maior de espécies comparativamente a outros estudos, o que se pode inferir grande conhecimento tradicional empregado na constituição e diversificação desses sistemas de uso da terra no local de estudo, considerando, o baixo nível tecnológico e força de trabalho familiar empregados. Assim, esses espaços característicos das paisagens rurais dos agricultores familiares do nordeste paraense correspondem elementos fundamentais dos seus agroecossistemas, gerando produção e garantindo a reprodução das famílias.

Palavras-chaves: sistemas agroflorestais, reprodução socioeconômica, desenvolvimento comunitário.

Abstract: The homegardens are common among family farmers in the Amazon, and sustainable land use systems developed from traditional knowledge. Besides having as main purpose the promotion of food security, they are spaces for socialization and recreation for farmers and can contribute to the composition of its income from the sale of production surplus. The aim of this study was to identify the diversity of yards from the flora and fauna composition. The research studied 37 homegardens in five communities in Igarapé-Açu and Marapanim, northeast Pará, using direct observation techniques in the field, application of semi-structured questionnaires, ethnographic notes and guided tour in homegardens. Were identified 107 plant species and 12 animals. These data show that there is a greater number of species compared to other studies, which can be inferred large traditional knowledge used in the creation and diversification of these land use systems in the study site, considering the low technological level and family labor employees. Thus, these characteristic spaces of the countryside of family farmers in northeast Pará match key elements of their agricultural ecosystems, generating production and ensuring the reproduction of families.

Key words: agroforestry systems, socioeconomic reproduction, community development.

\footnotetext{
*Autor para correspondência

Recebido para publicação em 05/10/2015; aprovado em 20/12/2015

${ }^{1}$ Eng. Florestal. Mestre em Agriculturas Familiares e Desenvolvimento Sustentável pelo Núcleo de Estudos sobre Agricultura Familiar - NEAF da Universidade Federal do Pará - UFPA, Campus Universitário do Guamá, Rua Augusto Corrêa, № 1, NEAF/UFPA, CEP: 66075-110, Belém-PA. E-mail: amandapquaresma@hotmail.com;

${ }^{2}$ Socióloga. Professora do ISARH/UFRA, Avenida Presidente Tancredo Neves, № 2501 - Terra Firme, UFRA, CEP: 66.077-830. Telefone: (91) 32105140.

Belém-PA. E-mail: ruthpara@yahoo.com.br;

${ }^{3}$ Eng. Agrônoma. D. Sc., Professora do Programa de Pós Graduação em Administração da Universidade da Amazônia - UNAMA, Professora do Instituto Socioambiental e dos Recursos Hídricos - ISARH da Universidade Federal Rural da Amazônia - UFRA, Avenida Presidente Tancredo Neves, $N^{\circ} 2501$ - Terra Firme, UFRA, CEP: 66.077-830. Telefone: (91) 32105140. Belém-PA. E-mail: cyntiamei@ hotmail.com;

${ }^{4}$ Eng. Agrônomo. D. Sc., Professor do NEAF/UFPA, Pesquisador da Embrapa Amazônia Oriental - CPATU, Travessa Doutor Enéas Pinheiro, s/n - Marco, EMBRAPA-CPATU, CEP: 66095-903. Telefone: (91) 32041014. Belém-PA. E-mail: okato@cpatu.embrapa.br
} 


\section{INTRODUÇÃO}

O conhecimento tradicional das populações da Amazônia foi historicamente construído por comunidades indígenas, ribeirinhas e caboclas, para garantir, principalmente, sua subsistência. Segundo Neves (2013), em todas as regiões tropicais do mundo, as populações locais desenvolveram sistemas integrados de domesticação de espécies, que asseguraram a manutenção da fertilidade do solo na produção de alimentos e de outros produtos, integrando a conservação ambiental ao processo produtivo por meio da diversidade biológica.

Cabe frisar que esse modo de produção tem sido cada vez mais reconhecido como um dos elementos centrais para o desenvolvimento e bem estar da humanidade e grande responsável pelo equilíbrio ambiental global (OLIVEIRA JÚNIOR; CABREIRA, 2012).

Sistemas integrados de produção, também chamados de Sistemas Agroflorestais (SAF), são sistemas de uso da terra amplamente utilizados em todo o mundo para a produção sustentável, combinando elementos agrícolas e florestais e, associando espécies vegetais e pequenos animais no mesmo espaço e/ou tempo. O SAF procura aumentar a produção de forma contínua utilizando práticas de manejo compatíveis com a cultura das populações locais (FARRELL; ALTIERE, 2012).

Comparados às monoculturas agrícolas e/ou florestais, Farrell e Altieri (2012) e Oliveira Júnior e Cabreira (2012) consideram que esses sistemas apresentam diversas vantagens tanto ecológicas quanto econômicas, fazendo uso mais eficiente dos recursos naturais pela interação entre os elementos, diminuindo os danos de degradação ambiental, além de influenciar positivamente o microclima e a hidrologia.

Ademais apresentam funções produtivas de inúmeros bens para seres humanos e animais, devido à colheita de cultivos diferentes ao longo do ano, combinando espécies arbóreas lenhosas, tanto frutíferas quanto madeireiras, com cultivos agrícolas e/ou animais, simultaneamente, o que propicia ao agricultor uma renda distribuída de forma mais homogênea e reduz a compra de insumos (FARRELL; ALTIERI, 2012; OLIVEIRA JÚNIOR; CABREIRA, 2012).

Em regiões tropicais como na Amazônia, um bom exemplo de SAF são os quintais agroflorestais. Oliveira et al. (2015) definem os quintais como a área próxima da casa, considerando que os agricultores mensuram e delimitam este espaço em seus lotes até 100 metros ao redor da residência. Nele estão os criadouros de pequenos animais, frutas, horta, plantas medicinais e até produtos comercializados. Segundo Farrell e Altiere (2012) sua estrutura é semelhante às florestas tropicais apresentando estratos e diversas espécies, podendo chegar a mais de 100 espécies por quintal.

O quintal agroflorestal também é conhecido como terreiro, miscelânea, horta familiar ou simplesmente quintal, e representa um elemento muito comum na paisagem do interior amazônico com suas casas simples, mas bem cercadas de verde. Porém, mesmo sendo frequentes, segundo Winklerprins e Souza (2005), os quintais agroflorestais possuem seu valor produtivo e reprodutivo ainda subestimado comparativamente ao modelo de agricultura industrial.

Paralelamente, os estudos sobre quintais agroflorestais vêm crescendo conforme Lourenço et al. (2009); Bezerra et al. (2012) e OLIVEIRA et al. (2015) devido à maior visibilidade dada a esses sistemas diversificados que abastecem os mercados locais, manejados com baixo nível tecnológico e adaptado às populações locais, contribuindo para a conservação da biodiversidade.

Para Winklerprins e Souza (2005) e Oliveira et al. (2015), os quintais são meios de subsistência local de múltiplos usos, que garantem a segurança alimentar, a sobrevivência das populações satisfazendo o abastecimento doméstico de alimentos básicos como fibras e proteínas, medicamentos naturais, materiais de construção e espaços de lazer, além de gerar alguma renda.

Eles asseguram um fluxo pequeno, mas contínuo de produtos, quando excedentes abastecem os mercados locais (WINKLERPRINS; OLIVEIRA, 2010). As espécies frutíferas são os principais produtos comercializados a partir dos quintais, pois, constituem a preferência das famílias quando planejam esse espaço.

Ainda segundo Winklerprins e Oliveira (2010), enquanto a roça é responsável por produzir gêneros de alto poder calorífico como mandioca, feijão e milho, o quintal fornece alimentos ricos em proteínas, vitaminas e sais minerais provenientes da diversidade de frutas e das criações dos pequenos animais, sendo as espécies frutíferas o principal produto comercializado a partir dos quintais, pois, constituem a preferência das famílias quando planejam esses espaços.

Quanto à implantação e o manejo desses espaços, eles são executados com emprego da força de trabalho familiar. Porém, são as mulheres as grandes responsáveis pela sua formação e manutenção, participando ativamente de todas as fases do quintal e assumindo sua condução. Vale frisar que as trocas entre os produtores, o conhecimento adquirido através da observação da natureza e a transmissão do conhecimento tradicional contribuem para sua diversificação (OLIVEIRA et al., 2015).

Dessa feita constitui uma parte importante da biodiversidade e engloba todos os elementos que interagem na produção agrícola: os espaços cultivados ou utilizados para criação de animais domésticos, as espécies direta ou indiretamente manejadas, como as cultivadas e seus parentes silvestres, e a diversidade genética a eles associada (SANTILLI, 2009).

Nesse sentido, o trabalho tem como objetivo identificar a diversidade dos quintais, a partir da composição de flora e fauna, buscando sistematizar as principais categorias de espécies, seu uso, manejo e divisão de tarefas.

\section{MATERIAL E MÉTODOS}

O trabalho foi desenvolvido no período de agosto de 2012 a fevereiro de 2014, em cinco comunidades de agricultores familiares dos municípios de Igarapé-Açu, com coordenada geográfica de $1^{\circ} 07^{\prime} 33^{\prime}$ ' de latitude sul e $47^{\circ} 37^{\prime}$ $27^{\prime}$ ' de longitude a oeste de Greenwith e, Marapanim que está localizado a $00^{\circ} 43^{\prime} 03^{\prime \prime}$ de latitude sul e $47^{\circ} 41^{\prime} 59^{\prime \prime}$ de longitude a oeste. As comunidades estudadas foram São João, Nossa Senhora do Rosário, Novo Brasil/Aparecida, Nova Olinda e Arsênio.

A pesquisa classifica-se como estudo descritivo, com abordagem qualitativa e quantitativa, desenvolvida por meio de pesquisa in loco, sendo realizada em 37 quintais 
agroflorestais com a contribuição de 73 pessoas entrevistadas no total.

Igarapé-Açu e Marapanim pertencem à mesorregião do nordeste paraense e à microrregião bragantina. O primeiro é distante $120 \mathrm{~km}$ da capital Belém, apresentando população total de 35. 887 habitantes, sendo 21.207 na zona urbana e 14.680 na zona rural. Já Marapanim fica a 142 km de Belém e apresenta população total de 26.602 habitantes, sendo 14.901 na zona rural e 11.704 na zona urbana (IBGE, 2010).

A região é caracterizada por uma agricultura itinerante que utiliza a prática de corte e queima, portanto, os ecossistemas são áreas alteradas com vegetação predominante de floresta secundária. A bacia hidrográfica possui diversos rios e igarapés, muitos apresentando assoreamento e poluição. A topografia atesta relevo simples e o clima é megatérmico úmido com temperatura média em torno de $25^{\circ} \mathrm{C}$ (IBGE, 2010).

Para obtenção das informações foram utilizados os métodos da pesquisa bibliográfica, observação direta em campo, aplicação de questionários semiestruturados, notas etnográficas e turnê guiada nos quintais agroflorestais com finalidade de inventariar as espécies de flora e fauna presentes.

Para identificação das espécies vegetais foram recolhidas amostras de materiais e enviadas ao herbário da Universidade Federal Rural da Amazônia - UFRA. Posteriormente foi utilizado o sistema APG III de taxonomia vegetal. Também foram consultados dois endereços eletrônicos, um nacional e outro internacional, para correta classificação de acordo com os autores, Flora do Brasil e Trópicos.

O questionário foi baseado na descrição da identificação da família (idade, sexo e escolaridade), trabalho e renda, organização social, flora e fauna presentes na comunidade, finalidades de uso do quintal, da flora e da fauna, manejo e calendário agrícola.

Os dados quantitativos foram organizados para análise usando o aplicativo Microsoft Excel. As análises foram realizadas por meio de métodos da estatística descritiva, com auxílio de representações gráficas e tabelas.

\section{RESULTADOS E DISCUSSÃO}

A idade média dos entrevistados foi de 51 anos, sendo 49 anos para as mulheres e 52 anos para os homens. Além do conhecimento empírico e da experiência acumulada, estes entrevistados se encontram em idade ativa para o desenvolvimento de atividades econômico-produtivas, portanto podem ser a base para o desenvolvimento local. O grau de escolaridade variou entre a $4^{\mathrm{a}}$ série do ensino fundamental para as mulheres e $5^{\mathrm{a}}$ série do ensino fundamental para os homens, com registro de casos de analfabetismo com maior frequência nas mulheres.

Esta situação se soma aos dados do Instituto Brasileiro de Geografia e Estatística (IBGE, 2010a), de que o estado do Pará possui 23.645 mulheres a frente de estabelecimentos rurais, sendo quase $20 \%$ destas analfabetas.
Quanto ao acesso a recursos externos, observou-se que $80 \%$ das famílias recebem algum tipo de benefício da previdência, principalmente, o Bolsa Família, enquanto 36\% dos entrevistados acessam a aposentadoria rural, contribuindo significativamente na composição da renda familiar.

Ademais, o trabalho fora da propriedade é realizado por $37 \%$ das mulheres entrevistadas, geralmente presentes no quadro de educação e saúde rural. Quanto aos homens, 25\% deles realizam trabalho externo à propriedade, geralmente se empregando como trabalhadores rurais em fazendas da região.

Piccin (2012) aponta que a agricultura familiar, visando complementar a renda, é pressionada a reforçar ou lançar mão de outras estratégias, além da diversificação produtiva, a despeito de atividades não agrícolas e a terceirização de mão de obra. Isto decorre da perda de competitividade do agricultor, dos menores preços pagos aos produtos agrícolas, das mudanças de mercado e de outras questões como a modernização da agricultura.

Estes fatores forçam os agricultores a inserirem-se em outras atividades, nos quais os processos de concorrência são menos intensos, tornando-se assim, uma forma de resistência no meio rural e reprodução das famílias (PICCIN, 2012; ESCHER et al., 2014), sendo este fenômeno conhecido como pluriatividade, que cada vez mais tem se tornado comum no mundo rural.

Segundo Escher et al. (2014), a pluriatividade é definida pela combinação entre o trabalho agrícola e as atividades fora da propriedade. Esta não apresenta um fenômeno inteiramente novo, uma vez que é sabido que os agricultores sempre mantiveram a combinação em múltiplas ocupações.

A participação em organizações políticas, como associações, é de apenas 33\% para as mulheres, enquanto que 97\% dos homens entrevistados são ativos nesses espaços. Estes dados são diferentes dos encontrados por Oliveira et al. (2015) onde cerca de 50\% das entrevistadas estava vinculada a alguma organização.

A participação em capacitações é menor ainda entre as mulheres, onde apenas $27 \%$ tiveram essa oportunidade em contraste com $79 \%$ dos homens entrevistados. Esta situação é preocupante considerando a relevância do papel da mulher nos quintas.

Aguiar e Rosa (2008) em pesquisa realizada em Santa Catarina constataram que os sistemas de produção mais diversificados tendem a estimular um processo de gestão menos centralizado pelo homem e mais participativo entre os gêneros, comparativamente àqueles baseados em um único tipo de exploração.

Sem dúvida, os aspectos socioeconômicos das famílias influenciam a constituição dos quintais, como por exemplo, a escolha das espécies, o tamanho, a divisão de tarefas e o manejo do quintal. Assim, pode-se compreender o quintal agroflorestal como uma extensão da casa e da própria família.

A composição florística constou da identificação de 107 espécies (Tabela 1), sendo esse valor superior aos relatados por Lourenço et al. (2009), que registrou 70 espécies vegetais em estudo realizado em quintais de três assentamentos na Amazônia Central. 
Tabela 1 - Espécies vegetais identificadas nos quintais agroflorestais das comunidades estudas. Pará, 2013.

\begin{tabular}{|c|c|c|c|}
\hline Nome Comum & Nome Científico & Família & Finalidade \\
\hline Abacate & Persia americana Mill. & Lauraceae Juss. & Alimentação \\
\hline Abacaxi & Ananas comosus hort. ex Descourt. & Bromeliaceae A. Juss. & Alimentação \\
\hline Abiu & Pouteria caimito (Ruiz \& Pav.) Radlk. & Sapotaceae Juss. & Alimentação \\
\hline Abricó & Mammea americana $\mathrm{L}$. & $\begin{array}{l}\text { Calophyllaceae J. } \\
\text { Agardh }\end{array}$ & Alimentação \\
\hline Acácia & $\begin{array}{l}\text { Cassia speciosa } \\
\text { Schrad. }\end{array}$ & Fabaceae Lindl. & Madeira \\
\hline Açaí & Euterpe pleraceae Mart. & Arecaceae Schultz Sch. & Alimentação \\
\hline Acerola & Malpighia punicifolia $\mathrm{L}$. & Malpighiaceae Juss. & Alimentação \\
\hline Ajirú & Chrysobalanus icaco $\mathrm{L}$ & Chrysobalanaceae R. Br. & Alimentação \\
\hline Alamanda & Allamanda cathartica $\mathrm{L}$. & Apocynaceae Juss. & Ornamentação \\
\hline Alfavaca & Ocimum basilicum var. pilosum (Willd.) Benth. & Lamiaeceae Martinov & Alimentação \\
\hline Ameixa & Prunus myrtifolia (L.) Urb. & Rosaceas Juss. & Alimentação \\
\hline Amor crescido & Portulaca pilosa $\mathrm{L}$. & Portulacaceae Juss. & Ornamentação \\
\hline Anador & Justicia pectoralis Jacq. & Acanthaceae Juss. & Medicamento \\
\hline Andiroba & Carapa guianensis Aubl. & Meliaceae A. Juss. & Madeira \\
\hline Anil & Indigofera domingensis Spreng. ex DC. & Fabaceae Lindl. & Medicamento \\
\hline Araticum & Annona crassiflora Mart. & Annonaceae Juss. & Medicamento \\
\hline Arruda & Ruta graveolens $\mathrm{L}$. & Rutaceae A, Juss. & Medicamento \\
\hline Babosa & Aloe arborescens Mill. & $\begin{array}{l}\text { Xanthorrhoeaceae } \\
\text { Dumort. }\end{array}$ & Medicamento \\
\hline Bacaba & Oenocarpus bacaba Mart. & Arecaceae Schultz Sch. & Alimentação \\
\hline Bacabi & Oenocarpus minor Mart. & Arecaceae Schultz Sch. & Alimentação \\
\hline Bacuri & Platonia insignis Mart & Clusiaceae Lindl. & Alimentação \\
\hline Banana & Musa paradisíaca $\mathrm{L}$. & Musaceae Juss. & Alimentação \\
\hline Bastão do imperador & Etlingera elatior (Jack) R.M. Sm. & Zingiberaceae Martinov & Ornamentação \\
\hline Biribá & Rollinia mucosa (Jacq.) Bail & Annonaceae Juss. & Alimentação \\
\hline Boldo & Plectranthus barbatus Andr. & Lamiaceae Martinov & Medicamento \\
\hline Cabi & $\begin{array}{c}\text { Banisteriopsis caapi (Spruce ex Griseb.) c.v } \\
\text { Morton }\end{array}$ & Malpighiaceae Juss. & Medicamento \\
\hline Cacau & Theobroma cacao L. & Malvaceae Juss. & Alimentação \\
\hline Café & Coffea arabica $\mathrm{L}$. & Rubiaceae Juss. & Alimentação \\
\hline Cajarana & Spondias monbin $\mathrm{L}$. & Anacardiaceae R. Br. & Alimentação \\
\hline Cajú & Anacardium ocidentale $\mathrm{L}$. & Anacardiaceae R. Br. & Alimentação \\
\hline Canarana & Hymenachne amplexicaulis (Rudge) Nees & Poaceae Barnhart & Medicamento \\
\hline Canela & Cinnamomum Zeylanicum Blume & Lauraceae Juss. & Alimentação \\
\hline Capim santo & Cymbopogon citratus (DC.) Stapf & Poaceae Barnhart & Medicamento \\
\hline Carambola & Averrhoa carambola $\mathrm{L}$. & Oxilidaceae R. Br & Alimentação \\
\hline Carrapateira & Ricinus communis $\mathrm{L}$. & Euphorbiaceae Juss. & Alimentação \\
\hline Castanha do Pará & Bertholletia excelsa Bonpl. & Lecythidaceae A. Rich. & Alimentação \\
\hline Catinga de mulata & Tanacetum vulgare $\mathrm{L}$. & $\begin{array}{l}\text { Asteraceae Bercht. \& J. } \\
\text { Presl }\end{array}$ & Medicamento \\
\hline Cedro & Cedrela odorata $\mathrm{L}$. & Meliaceae A. Juss. & Madeira \\
\hline Chicória & Chicoruim endívia $\mathrm{L}$. & $\begin{array}{l}\text { Asteraceae Bercht. \& J. } \\
\text { Presl }\end{array}$ & Alimentação \\
\hline Cipó de alho & Mansoa Alliaceae L. & Bignoniaceae A. Juss. & Medicamento \\
\hline Coco & Cocus nucifera $\mathrm{L}$. & Arecaceae Schultz Sch. & Alimentação \\
\hline Coramina & Pedilanthus tithymaloides Poit. & Euphorbiaceae Juss. & Medicamento \\
\hline
\end{tabular}




\begin{tabular}{|c|c|c|c|}
\hline Cravo & Dianthus caryophyllus L. & $\begin{array}{c}\text { Caryophyllaceae A. } \\
\text { Rich. }\end{array}$ & Ornamentação \\
\hline Cuieira & Crescentia cujete $\mathrm{L}$. & Bignoniaceae Juss. & Utensílio \\
\hline Cupuaçú & Theobroma grandiflorum $\mathrm{L}$. & Malvaceae Juss. & Alimentação \\
\hline Elixir parigórico & Piper elogatum $\mathrm{R}$. et $\mathrm{P}$. & Piperaceae R. Br & Medicamento \\
\hline Erva cidreira & Melissa officinalis $\mathrm{L}$. & Lamiaceae Martinov. & Medicamento \\
\hline Eucalipto & Eucalyptus globus Labil & Myrtaceae Juss. & Madeira \\
\hline Genipapo & Genipa americana $\mathrm{L}$. & Rubiaceae Schultz Sch. & Alimentação \\
\hline Goiaba & Psidium guajava $\mathrm{L}$. & Myrtaceae Juss. & Alimentação \\
\hline Graviola & Annonamuricata $\mathrm{L}$. & Annonacaeae Lindl. & Alimentação \\
\hline Guarumã & Ischnosiphon arouma Koern. & Marantaceae Juss. & Artesanato \\
\hline Helicônea & Heliconia rostrata $\mathrm{L}$. & Heliconiaceae Barnhart & Ornamental \\
\hline Hortelã & Mentha ssp.L. & Lamiaceae Juss. & Medicamento \\
\hline Inajá & Maximiliana Maripa Aublet Drude & Arecaceae Schultz Sch. & \\
\hline Ingá & Inga edulis Mart & Fabaceae A. Rich. & Alimentação \\
\hline Insulina & Cissus siciyoides $\mathrm{L}$. & Vitaceae A. Juss. & Medicamento \\
\hline Ipê & Handroanthus spp.L. & Bignoniaceae Juss. & Madeira \\
\hline Jaca & Artocarpus heterophylla Lam. & Moraceae A. Juss. & Alimentação \\
\hline Jambo & Syzygium jambos L. Alston & Myrtaceae Juss. & Alimentação \\
\hline Japana & Ayapana triplinervis $\mathrm{L}$. & Asteraceae A. Juss & Medicamento \\
\hline Jarana & Holopixidium jarana Ducke & Lecythidaceae A. Rich. & Madeira \\
\hline Jerimum & Cucurbita spp.L. & Cucurbitaceae Juss. & Alimentação \\
\hline Jucá & Caesalpinia ferrea Mart. & Caesalpiniaceae R. Br. & Medicamento \\
\hline Laranja & Citrus sinensis Persoon. & Rutaceae Lindl. & Alimentação \\
\hline Limão & Citrus limon (Christ.) Swingle & Rutaceae Lindl. & Alimentação \\
\hline Macaxeira & Manihot ssp L. & Euphorbiaceae Giseke & Alimentação \\
\hline Mamão & Carica papaya $\mathrm{L}$. & Caricaceae Juss. & Alimentação \\
\hline Mamona & Ricinus comunis $\mathrm{L}$. & Euphorbiaceae Juss. & Alimentação \\
\hline Mandioca & Manihot esculenta $\mathrm{L}$. & Euphorbiaceae Juss. & Alimentação \\
\hline Manga & Mangfera indica $\mathrm{L}$. & Anacardiaceae R. Br. & Alimentação \\
\hline Manjericão & Ocimum basilicum $\mathrm{L}$. & Lamiaeceae Martinov. & Alimentação \\
\hline Manjerona & Origanum majorana $\mathrm{L}$. & Lamiaceae Martinov & Alimentação \\
\hline Maracujá & Passiflora edulis Sims & Passifloraceae Juss. & Alimentação \\
\hline Marupaúba & Simarouba amara $\mathrm{L}$. & Simarubaceas Juss. & Madeira \\
\hline Mastruz & Chenopodium ambrosioides $\mathrm{L}$. & Chenopodiaceae Juss. & Medicamento \\
\hline Mini-ixora & Ixora coccinea $\mathrm{L}$. & Rubiaceae Juss. & Ornamentação \\
\hline Mogno & Swieteniamacrophylls King & Meliaceae A. Juss. & Madeira \\
\hline Mucuracaá & Petiveria alliacea $\mathrm{L}$. & Phytolacaceae Juss. & Medicamento \\
\hline Muruci & Byrsonima crassifolia (L.) Kunth & Malpighiaceae Juss. & Alimentação \\
\hline Noni & Morinda citrifolia $\mathrm{L}$. & Rubiaceae Juss. & Medicamento \\
\hline Oiti & Licania tomentosa $\mathrm{L}$. & $\begin{array}{c}\text { Chrysobalanaceae A. } \\
\text { Juss. }\end{array}$ & Alimentação \\
\hline Oriza & Pogostemon patchouly Pellet. & Lamiaceae Martinov. & Medicamento \\
\hline Papoula (variada) & Hibiscus rosasinensis & Malvaceae Juss. & Ornamentação \\
\hline Paricá & Schizolobium amazonicum Huber ex Ducke & Fabaceae Lindl. & Madeira \\
\hline Pariri & Arrebidaea chica Verlot. & Bignoniaceae A. Juss. & Medicamento \\
\hline Pimenta & Piper nigrum $\mathrm{L}$. & Piperaceae Giseke & Alimentação \\
\hline Pinheiro & Araucaria angustifólia & Araucareacaeae Juss. & Madeira \\
\hline Piquiá & Caryocar villosum (Aubl.) Pers. & Caryocaraceae A. Rich. & Alimentação \\
\hline
\end{tabular}




$\begin{array}{cc}\text { Pupunha } & \text { Bactris gasipaes } \text { Kunth } \\ \text { Quina } & \text { Geissospermum laeve Baill } \\ \text { Sabugueiro } & \text { Sambucus australis } \text { Chamisso et Schlechtenda } \\ \text { Samambaia } & \text { Nephrolepis exaltada } \text { L. } \\ \text { Seringueira } & \text { Hevea brasiliensis } \text { Muell. Arg. } \\ \text { Sucurijú } & \text { Mikania lidleyana } \text { Dc. } \\ \text { Sucuúba } & \text { Himathanthus } \text { sucuuba } \text { (Spruce) Woods } \\ \text { Tangerina } & \text { Citurs reticulada } \text { Blanco } \\ \text { Taperebá } & \text { Spondias lutea } \text { L. } \\ \text { Tauari } & \text { Couratari cf. } \text { oblongifolia } \text { Ducke } \\ \text { Teca } & \text { Tectona grandis } \text { L. } \\ \text { Titônia } & \text { Thitonia diversifolia } \text { L. } \\ \text { Trevo } & \text { Trifolium repens } \text { L. } \\ \text { Tucumã } & \text { Astrocaryum tucuma } \text { Burret } \\ \text { Ucuúba } & \text { Virola } \text { surinamensisi } \text { L. } \\ \text { Urucum } & \text { Bixa orellana } \text { L. } \\ \text { Vindicá } & \text { Alpinia } \text { cf. } \text { nutans } \text { Posc. } \\ \text { Visgueiro } & \text { Parkia pendula } \text { Bent. }\end{array}$

$\begin{array}{cc}\text { Arecaceae Schultz Sch. } & \text { Alimentação } \\ \text { Apocynaceae Juss. } & \text { Medicamento } \\ \text { Adoxaceae Juss. } & \text { Ornamentação } \\ \text { Davaliacaeae Juss. } & \text { Ornamentação } \\ \text { Euphorbiaceae R. Br. } & \text { Madeira } \\ \text { Asteraceae Giseke } & \text { Medicamento } \\ \text { Apocynaceae Juss. } & \text { Madeira } \\ \text { Rutaceae A, Juss. } & \text { Alimentação } \\ \text { Anacardiaceae R. Br. } & \text { Alimentação } \\ \text { Lecythidaceae A. Rich. } & \text { Madeira } \\ \text { Verbenaceae Juss. } & \text { Madeira } \\ \text { Asteraceae A. Juss } & \text { Ornamentação } \\ \text { Fabaceae Lindl. } & \text { Ornamentação } \\ \text { Arecaceae Schultz Sch. } & \text { Alimentação } \\ \text { Miristaceaea Juss. } & \text { Madeira } \\ \text { Bixaceaea A. Juss. } & \text { Alimentação } \\ \text { Zingiberaceae Juss. } & \text { Medicamento } \\ \text { Fabaceae Lindl. } & \text { Alimentação }\end{array}$

Fonte: Dados da pesquisa com descrição das famílias e espécies a partir dos sites www.tropicos.org e floradobrasil.jbrj.gov.br

De acordo com a Figura 1, as plantas medicinais somaram $37 \%$ do total identificado, sendo o maior grupo observado. Segundo Winklerprins e Souza (2005), elas são cultivadas com maior frequência em áreas onde o acesso a postos de saúde e farmácias é mais difícil, já que é um custo oneroso para a família tirar de seu orçamento o valor de compra dos remédios industrializados. Para tratar as doenças, as plantas são consumidas, principalmente, sob as formas de infusões, conhecidas como "banhos", e chás que proporcionam alívio para dores e outras enfermidades.
Resultados semelhantes foram encontrados por Ming e Amaral Júnior (2005) e Lima et al. (2011) na Amazônia e Cunha e Bortolotto (2011) em Mato Grosso, todos com plantas medicinais. Bezerra et al. (2012) constataram que $71,14 \%$ da comunidade de mimoso no município de Paulista na Paraíba extraiam as plantas medicinais do quintal de suas casas.

Figura 1. Categorias de espécies identificadas nos quintais agroflorestais. Pará, 2013.

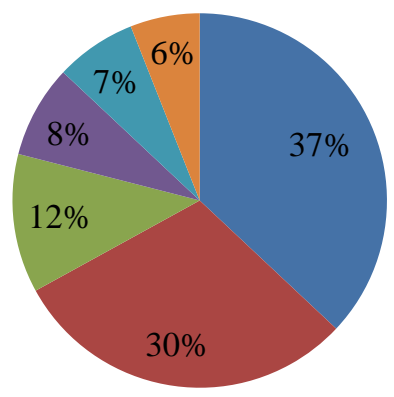

Medicinais

- Frutíferas/alimentícias

- Madeireiras

- Ornamentais

- Codimentares

- Outras

Fonte: Dados da pesquisa

O conhecimento sobre cada planta, a indicação e a forma de uso é repassado entre as gerações de uma mesma família ou entre famílias, principalmente, pelas mulheres. A homeopatia é um recurso muito importante para a saúde e bem estar e o compartilhamento desses saberes reforça a importância dos conhecimentos tradicionais das populações rurais (WINKLERPRINS; SOUZA, 2005).

As plantas frutíferas, que agregaram outras alimentícias em seu grupo, somaram $30 \%$ do total de espécies identificadas. Cinco espécies foram mais frequentes, respectivamente: banana, coco, cupuaçu, açaí e limão e, apesar de terem sido o segundo maior grupo pelo número de espécies, apresentaram maior número de indivíduos nos quintais, ou seja, maior frequência.

A preferência por espécies frutíferas, segundo Santilli (2009), está relacionada à alimentação das famílias, pois são alimentos frescos de acesso imediato, diferente dos alimentos comprados que não se conhece o tempo e modo de armazenamento e circulação, além do próprio processo produtivo, onde não se sabe dos teores de agrotóxicos. Além 
disso, são espécies que possuem valor comercial e que podem contribuir na renda familiar, assim como proporcionar conforto ambiental e lazer para a família.

Plantas madeireiras, entre nativas e exóticas cultivadas, somaram $12 \%$ do total de espécies identificadas. Geralmente são espécies nativas, "deixadas" na propriedade após a limpeza ou mesmo mudas que foram doadas por entidades de pesquisa em projetos de conservação da biodiversidade. Quando apresentam baixo valor comercial são utilizadas em demandas da família, como lenha e madeira para construções mais rústicas, sendo mais comum do que a comercialização de espécies nobres, com maior valor de mercado, pois essas espécies são vistas como uma "poupança" a ser utilizada somente quando houver grande necessidade.

Plantas ornamentais representam $8 \%$ do total de espécies identificadas. Porém, deve-se destacar que, dentre as principais espécies encontradas, há variedade de indivíduos a partir de uma mesma espécie que foram "enxertados" com algumas características diferentes nos levando a considerar a possibilidade de espécies novas que sequer foram catalogadas.
Essa grande variedade de plantas ornamentais pode ser explicada, segundo Winklerprins e Souza (2005), pelo prazer estético e significado simbólico que seu cultivo representa, principalmente, para as mulheres, que são quase que exclusivamente as cuidadoras diretas dessa categoria encontrada nos quintais.

Plantas utilizadas como condimentos alimentares somaram $7 \%$ do total de espécies identificadas. Segundo Winklerprins e Souza (2005), elas são adicionadas às refeições para dar mais sabor a pratos regionais como peixes, frangos e carnes em geral. A categoria "outras", representando $6 \%$ do total de espécies identificadas, agregam plantas utilizadas para diversas finalidades, como artesanato e utensílios domésticos.

São componentes comuns de sistemas integrados de produção, as espécies animais. Dentre as presentes nos quintais agroflorestais pesquisados, têm-se 12 espécies no total, sendo 2 de animais domésticos e 10 de animais domesticados, a maioria de pequeno porte conforme a Figura 2.

Figura 2. Número de quintas com presença de animais domésticos e domesticados. Pará, 2013.

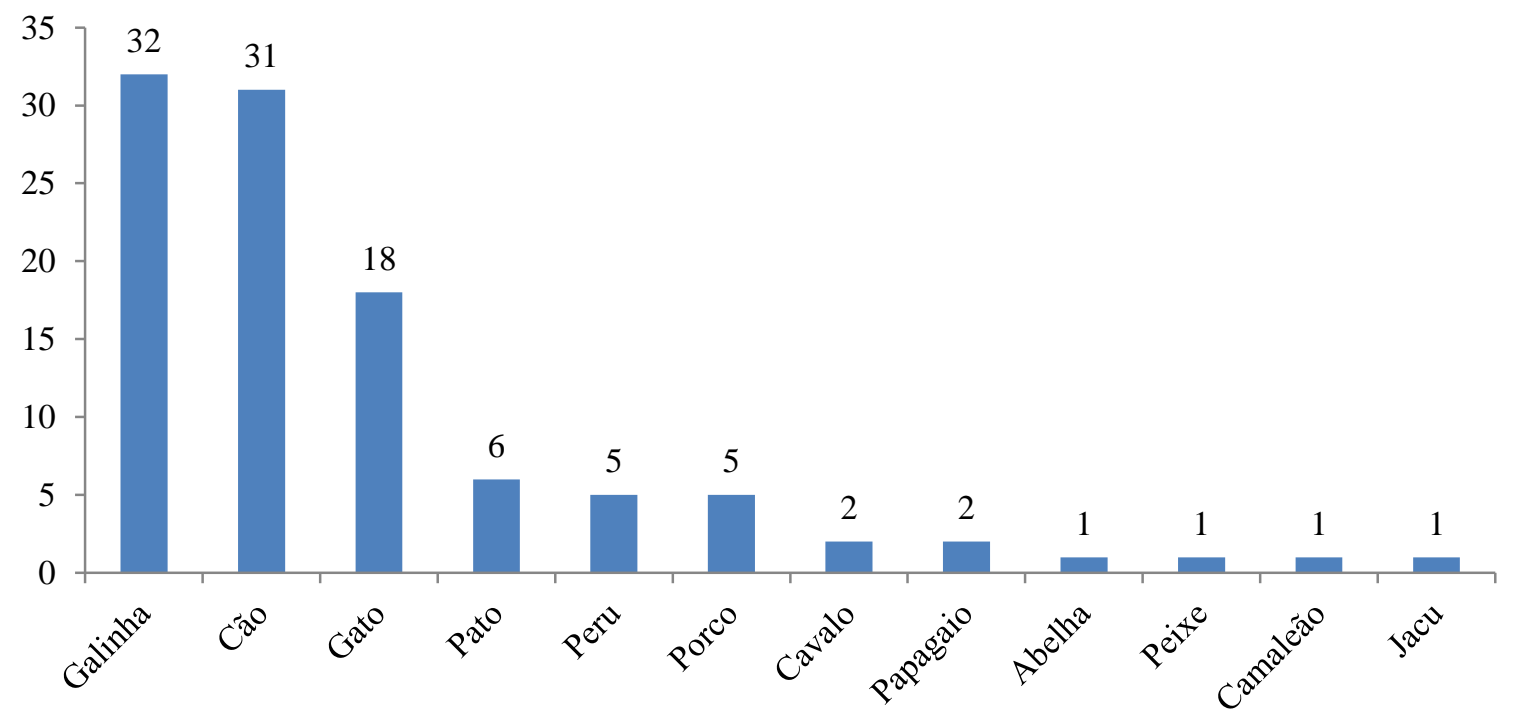

Fonte: Dados da pesquisa

As aves, porco e peixe têm a finalidade principal de complementação da dieta familiar através do consumo. Dentre estes, a galinha foi o animal domesticado mais comum, presente em 32 dos 37 quintais agroflorestais pesquisados. A despeito de serem principalmente para 0 consumo próprio, garantindo a segurança alimentar, também podem ser comercializadas quando não prejudicam o consumo. Também geram os ovos, produtos muito utilizados na dieta alimentar das famílias e que possui venda rápida e fácil nos mercados locais. Vale frisar que estes dados corroboram com Pereira et al. (2010) que dentre as espécies animais encontraram maior número de aves nos quintais do assentamento Belo Horizonte I em São Domingos do Araguaia, Pará.

De acordo com as famílias, o menor número de criações de pato, peru, porco e peixe se deve, principalmente, aos custos maiores com alimentação comparativamente às galinhas, daí o motivo de maior preferência de criação por aquela ave.
Os cavalos são utilizados para diminuir o esforço físico no trabalho agrícola, já a criação de abelhas contribui para a renda familiar através da venda de mel e própolis. Os cães e gatos, segundo relatado pelas famílias, são importantes para companhia e lazer que esses animais proporcionam, além da segurança, no caso dos cães, pois tem sido comum a prática de furtos nas propriedades e, os gatos, que também afugentam ratos, aranhas e cobras.

Apesar de cães e gatos também serem considerados membros da família, na maioria dos casos, não é comum circularem no interior das residências, ficando seu espaço reservado nos quintais.

Todos os animais observados geram benefícios diretos ou indiretos paras as famílias, seja na alimentação, no aumento da renda familiar, no trabalho realizado ou mesmo no lazer proporcionado e são considerados pelas famílias elementos importantes dentro dos agroecossistemas.

Essa diversificação de produtos e serviços gerados pelos quintais agroflorestais permite uma segurança alimentar e 
financeira para a família, pois garante produção variada ao longo do ano. A Tabela 2 mostra a distribuição dos principais produtos dos quintais agroflorestais ao longo dos meses do ano.

Tabela 2 - Calendário de produção das principais espécies identificadas nos quintais agroflorestais distribuídas ao longo do ano. Pará, 2013.

\begin{tabular}{|c|c|c|c|c|c|c|c|c|c|c|c|c|}
\hline $\begin{array}{c}\text { MESES/ } \\
\text { ESPÉCIES }\end{array}$ & $\mathrm{J}$ & $\mathrm{F}$ & M & A & M & $\mathrm{J}$ & $\mathbf{J}$ & A & $\mathrm{S}$ & $\mathrm{O}$ & $\mathrm{N}$ & $\mathrm{D}$ \\
\hline Açaí & & & & & & & $\mathrm{X}$ & $\mathrm{X}$ & $\mathrm{X}$ & $\mathrm{X}$ & $\mathrm{X}$ & $\mathrm{X}$ \\
\hline Banana & $X$ & $\mathrm{X}$ & $X$ & $X$ & $\mathrm{X}$ & $X$ & $X$ & $X$ & $\mathrm{X}$ & $\mathrm{X}$ & $\mathrm{X}$ & $\mathrm{X}$ \\
\hline Cacau & $X$ & $X$ & $X$ & $X$ & $\mathrm{X}$ & $\mathrm{X}$ & $X$ & $X$ & $X$ & $\mathrm{X}$ & $X$ & $\mathrm{X}$ \\
\hline Caju & & & & & & $X$ & $X$ & $X$ & $X$ & $X$ & $X$ & $\mathrm{X}$ \\
\hline Coco & $X$ & $\mathrm{X}$ & $X$ & $X$ & $\mathrm{X}$ & $X$ & $X$ & $\mathrm{X}$ & $X$ & $X$ & $X$ & $X$ \\
\hline Cupuaçu & $X$ & $\mathrm{X}$ & $X$ & $\mathrm{X}$ & $\mathrm{X}$ & & & & & & & \\
\hline Goiaba & $X$ & $X$ & $X$ & $X$ & $X$ & $X$ & & & & & & \\
\hline Jambo & $X$ & $X$ & $X$ & $X$ & $\mathrm{X}$ & $X$ & & & & & & \\
\hline Laranja & $X$ & $X$ & $X$ & $X$ & $X$ & $X$ & $X$ & $X$ & $\mathrm{X}$ & $X$ & $X$ & $X$ \\
\hline Limão & $X$ & $\mathrm{X}$ & $\mathrm{X}$ & $X$ & $\mathrm{X}$ & $X$ & $X$ & $\mathrm{X}$ & $\mathrm{X}$ & $\mathrm{X}$ & $\mathrm{X}$ & $X$ \\
\hline Manga & $X$ & $X$ & & & & & & & & & $X$ & $X$ \\
\hline Ovos & $X$ & $X$ & $X$ & $X$ & $X$ & $X$ & $X$ & $X$ & $X$ & $X$ & $X$ & $X$ \\
\hline Proteína (carne) & $X$ & $X$ & $\mathrm{X}$ & $\mathrm{X}$ & $\mathrm{X}$ & $\mathrm{X}$ & $\mathrm{X}$ & $X$ & $\mathrm{X}$ & $\mathrm{X}$ & $\mathrm{X}$ & $\mathrm{X}$ \\
\hline Plantas condimentares & $\mathrm{X}$ & $\mathrm{X}$ & $\mathrm{X}$ & $\mathrm{X}$ & $\mathrm{X}$ & $\mathrm{X}$ & $X$ & $\mathrm{X}$ & $\mathrm{X}$ & $\mathrm{X}$ & $\mathrm{X}$ & $\mathrm{X}$ \\
\hline Plantas madeireiras & $\mathrm{X}$ & $\mathrm{X}$ & $\mathrm{X}$ & $\mathrm{X}$ & $\mathrm{X}$ & $\mathrm{X}$ & $\mathrm{X}$ & $\mathrm{X}$ & $\mathrm{X}$ & $\mathrm{X}$ & $\mathrm{X}$ & $\mathrm{X}$ \\
\hline Plantas medicinais & $\mathrm{X}$ & $\mathrm{X}$ & $\mathrm{X}$ & $\mathrm{X}$ & $\mathrm{X}$ & $\mathrm{X}$ & $\mathrm{X}$ & $\mathrm{X}$ & $\mathrm{X}$ & $\mathrm{X}$ & $\mathrm{X}$ & $\mathrm{X}$ \\
\hline Pupunha & $\mathrm{X}$ & $\mathrm{X}$ & $\mathrm{X}$ & $\mathrm{X}$ & $\mathrm{X}$ & & & & & & & \\
\hline
\end{tabular}

Fonte: Dados de pesquisa

A despeito de não serem processos planejados rigorosamente e empregarem baixo nível tecnológico, os quintais agroflorestais devem ser considerados como área de produção, devido alguns produtos estarem disponíveis ao longo de todo o ano.

Vale frisar que a implantação e o manejo dos quintais agroflorestais obedecem ao grau de importância das espécies para a subsistência e perspectiva de comercialização dos excedentes para a família. Também se leva em consideração a facilidade de cultivo, a intensidade do manejo e o tempo de retorno da produção (WINKLERPRINS; OLIVEIRA, 2010). Assim, nos quintais estudados é comum ao longo do ano, principalmente a disponibilidade de ovos, plantas medicinais, condimentares e ornamentais, frutas como banana, coco e limão, além da proteína proveniente das criações de animais, principalmente, as galinhas.

Queimar as folhas ainda é uma opção muito utilizada por dois motivos relatados pelos entrevistados: limpeza imediata do quintal proporcionando beleza estética e formação também quase imediata de adubo para as plantas. Porém, no decorrer das queimadas o solo vai empobrecendo de nutrientes e de vida microbiótica.

Assim, uma prática que vem crescendo entre as famílias entrevistadas é a compostagem, que além de aproveitar as folhas varridas do quintal, também agrega restos da alimentação e outros materiais produzidos no lote para passar por um processo de decomposição acelerada podendo disponibilizar nutrientes para plantas do quintal, do SAF e da horta sem adição de adubos minerais, diminuindo e/ou atenuando os custos com a manutenção da fertilidade do solo.
De forma geral, os quintais agroflorestais não apresentam uma organização espacial exata. Tendo em vista que a principal dificuldade esta em delimitar a área dos quintais, pois a própria família não se preocupa em estabelecer esse limite, separando o quintal da casa, da horta, do SAF ou mesmo da roça. O quintal muitas vezes acaba abrigando elementos e funções de todos os outros espaços. Nele é comum encontrar o banheiro de fossa seca, a cozinha a céu aberto com os famosos "jiraus" e fogões de lenha, a corda para estender e secar roupas, a casa para produção de farinha também conhecida como "retiro", a estrutura suspensa ou cercada que abriga a horta, assim como as mesas e bancos na sombra das árvores, além das crianças e animais correndo soltos pelo quintal.

Outro aspecto interessante do quintal agroflorestal está para além da satisfação das necessidades domésticas de primeiro gênero, pois eles também representam espaços de socialização e lazer das famílias entre si e com a comunidade. As crianças brincam correndo e subindo em árvores, os jovens e adultos descansam e conversam à sombra das árvores, mostrando que o quintal representa bem estar às famílias além de além de outras atividades da vida privada, ou seja, atividades que permitem a reprodução da força de trabalho e da própria vida no campo.

\section{CONCLUSÕES}

A maioria das espécies dos quintais agroflorestais pesquisados é utilizada diretamente pelas famílias, seja na alimentação, na prevenção e tratamento de enfermidades, 
estética da casa, ente outros, na forma in natura ou passando por processos primários de beneficiamento.

As espécies comestíveis promovem, principalmente, a segurança alimentar das famílias ao longo do ano e, contribuem para a composição da renda familiar pela comercialização do seu excedente. As plantas medicinais, as frutas e as galinhas "caipiras" são as principais espécies encontradas nos quintais agroflorestais.

Os quintais agroflorestais são componentes importantes na dinâmica das famílias, sendo uma extensão delas e de suas casas. No nordeste paraense ele cumpre papel fundamental na produção e reprodução da agricultura familiar amazônica.

\section{REFERÊNCIAS BIBLIOGRÁFICAS}

AGUIAR, V. V. P.; ROSA, E. P. da. Sistemas de produção e gênero na agricultura familiar. Fazendo Gênero (Florianópolis - SC), v. 8, agosto de 2008.

BEZERRA, A. M. F.; BEZERRA, K. K. S.; SOUSA, L. C. F. S.; SOUSA, J. S.; BORG, M. G. B. Plantas medicinais utilizadas pela comunidade de mimoso no município de Paulista, Paraíba - Brasil. Revista Verde (Mossoró RN), v. 7, n. 5, p. 06-11, dezembro de 2012 (Edição Especial).

CUNHA, S.A.; BORTOLLOTO, I.M. Etnobotânica de Plantas Medicinais no Assentamento Monjolinho, município de Anastácio, Mato Grosso do Sul, Brasil. Acta Botanica Brasilica, v. 25, n. 3, p. 685-698, 2011.

ESCHER, F.; SCHNEIDER, S.; SCARTON, L. M.; CONTERATO, M. A. Caracterização da Pluriatividade e dos Plurirrendimentos da Agricultura Brasileira a partir do Censo Agropecuário 2006. Revista de Economia e Sociologia Rural, v. 52, p. 643-667, 2014.

FARRELL, J. G.; ALTIERI, M. A. Sistemas agroflorestais. In: ALTIERI, M. Agroecologia: bases científicas para uma agricultura sustentável. São Paulo, Rio de Janeiro: Expressão Popular, AS-PTA, 2012. p. 281-304.

FLORA DO BRASIL 2020 EM CONSTRUÇÃO. Jardim Botânico do Rio de Janeiro. Disponível em: < http://floradobrasil.jbrj.gov.br/ > Acesso em: $18 \mathrm{de}$ março de 2014.

IBGE - Instituto Brasileiro de Geografia e Estatística. Censo Agropecuário, 2010a.

IBGE - Instituto Brasileiro de Geografia e Estatística. Cidades, 2010. Disponível em: <http://www.ibge.gov.br/home/pesquisa/igarape.gov.br \%2F\&ref=\&ss=5461j3212597j16>. Acesso: 23 de outubro 2012.

LIMA, P. G. C.; COELHO-FERREIRA, M. R.; OLIVEIRA, R. Plantas medicinais em feiras e mercados públicos do Distrito Florestal Sustentável da BR-163, Estado do Pará, Brasil. Acta Botanica Brasílica, v. 25, p. 422-434, 2011.
LOURENÇO, J. N. P.; SOUSA, S. G. A.; WANDELLI, E. V.; LOURENÇO, F. S.; GUIMARÃES, R. R.; CAMPOS, L. S.; SILVA, R. L.; MARTINS, V. F. C. Agrobiodiversidade nos Quintais Agroflorestais em Três Assentamentos na Amazônia Central. Revista Brasileira de Agroecologia, v. 4, n. 2, p. 965-969, 2009.

MING, L. C.; AMARAL JUNIOR, A. Aspectos Etnobotânicos de Plantas Medicinais na Reserva Extrativista "Chico Mendes": Florística e Botânica Econômica do Acre, Brasil. The New York Botanical Garden, 2005. Disponível em: http://www.nybg.org/bsci/acre/www1/medicinal.html. Acesso: 20 de maio de 2012.

NEVES, P. D. M. Sistemas agroflorestais como fomento para a segurança alimentar e nutricional. Revista Verde (Mossoró - RN - BRASIL), v. 8, n. 5, p. 199 -207, dezembro de 2013 (Edição Especial).

OLIVEIRA JÚNIOR, C. J. F.; CABREIRA, P. P. Sistemas agroflorestais: potencial econômico da biodiversidade vegetal a partir do conhecimento tradicional ou local. Revista Verde (Mossoró - RN - Brasil), v.7, n.1, p. 212224, janeiro-março de 2012.

OLIVEIRA, C. M.; SILVA, R. O.; ALMEIDA, R. H. C.Diversificação produtiva, reprodução socioeconômica e mulheres no assentamento periurbano Mártires de Abril - Pará. Nucleus, v.12, n.1, p. 253-266, abril de 2015.

PEREIRA, C. N.; MANESCHY, R. Q.; OLIVEIRA, P. D.; OLIVEIRA, I. K. de S. Caracterização de quintais agroflorestais no projeto de assentamento Belo Horizonte I, São Domingos do Araguaia, Pará. Agroecossistemas, v. 2, n. 1, p. 73-81, 2010.

PICCIN, M. B. Assentamentos rurais e geração de renda: posição social restringida, recursos socioculturais e mercados. Economia e Sociedade (Campinas - SP), v. 21, n. 1, p. 115-141, abril de 2012.

SANTILLI, J. F. R. Agrobiodiversidade e direitos dos agricultores. PUCviva Revista, v. 36, p. 46-55, 2009.

TRÓPICOS. Disponível em: http://www.tropicos.org/. Acesso em: 17 de março de 2014.

WINKLERPRINS, A. M. G. A.; OLIVEIRA, P. S. de S. Urban agriculture in Santarém, Pará, Brazil: diversity and circulation of cultivated plants in urban homegardens. Boletim Museu Paraense Emílio Goeldi. Ciências Humanas, Belém, v. 5, n. 3, p. 571-585, setdez, 2010.

WINKLERPRINS, A. M. G. A.; SOUZA, P. S. de. Surviving the City: Urban Home Gardens and the Economy of Affection in the Brazilian Amazon. Journal of Latin American geography, v. 4, n. 1, p. 107-126, 2005. 